\title{
Reliability of manual segmentation of cornea, contact lens and tear film using a high-resolution OCT
}

\author{
Patrícia Rodrigues $^{\mathrm{a}}$, Daniela Lopes-Ferreira ${ }^{\mathrm{a}}$, Andreia Catarino ${ }^{\mathrm{a}}$, Alejandro Cervino ${ }^{\mathrm{b}}$, Antonio Queirós ${ }^{\mathrm{a}}$ and \\ José Manuel González-Méijome ${ }^{\mathrm{a} * \text { (iD }}$ \\ ${ }^{a}$ Clinical \& Experimental Optometry Research Lab (CEORLab), Center of Physics, University of Minho, Braga, Portugal; \\ ${ }^{b}$ Optometry Research Group, Department of Optics, University of Valencia, Valencia, Spain
}

(Received 26 January 2014; accepted 2 December 2014)

\begin{abstract}
In this study, we aimed to evaluate the intra/inter-session and intra/inter-observer variability of manual segmentation of thickness of the pre-lens tear film, contact lens (CL), post-lens tear film, epithelium, bowman's layer, stroma and the whole cornea and CLs with a flat and a steep fit, using a commercial high-resolution spectral domain optical coherence tomographer (OCT). Two different observers obtained three repeated measures in two separate sessions. A high correlation was found between the values obtained by the two different observers, except for the thinner layers, epithelium, and Bowman's layer. Inter-observer analysis showed a high consistency in the measurements obtained by both observers $\left(r^{2} \geq 0.80 ; p<0.001\right)$ for the thicker layers: CL, stroma, and total cornea. Intra-observer analysis of measurements obtained by each observer within the same session displayed no statistically significant differences between the three repeated measures for both observers $(p>0.05)$. The present results suggest that manual segmentation of anterior segment OCT images in CL wearers provides acceptable levels of repeatability between observers and between different sessions for the thinner layers, while presenting a high level of repeatability for the thicker layers.
\end{abstract}

Keywords: optical coherence tomography; manual layer segmentation; repeatability; corneal thickness; epithelial thickness; tear film thickness

\section{Introduction}

The increasing number of contact lens (CL) materials, designs, and wear options over the last years make increasingly important to determine the intimate relationship between the ocular surface and the CL. The detailed assessment of such relationships might play an important role in our understanding of the comfort of CLs and the detailed evaluation of the cornea will be relevant for the detection and follow-up of corneal disease [1,2]. With all these features in mind, optical coherence tomography (OCT) has been established as a valuable instrument in clinical practice and research first for the retina and later to the anterior surface of the eye $[3,4]$.

OCT is a noninvasive, noncontact optical imaging modality, which applies low coherence interferometry and uses an image mapping process to display highresolution cross-sectional images of the ocular structure. Originally designed to measure the different layers of the retina [6,7], it has now assumed an increasingly important role in the diagnosis and management of a multitude of ocular conditions, including age-related macular degeneration, diabetic macular edema, and many other retinal conditions [8].

More recently OCT technology has expanded its applicability to measuring corneal and epithelial thick- ness in living corneas in situ [7,9,10]. Low coherence interferometry provides a single log reflectivity profile in which corneal which the thickness of the different corneal layers can be determined by measuring the distance of $\log$ reflectivity peaks from the various interfaces [9]. Typically, corneal thickness is determined as the distance between the first and last peak while epithelial thickness is determined by the distance between the first and second peak, as described in previous studies $[9,11]$.

Studies evaluating the corneal response to $\mathrm{CL}$ wear have recognized the potential of OCT [11], but the early technology based on time domain OCT had limitations in terms of the ability to obtain real-time imaging of the ocular surface and to carry out threedimensional imaging or capture motion artifacts. When compared with optical pachymetry, OCT presents an overestimation of thickness in normal and edematous corneas [11]. Recently, spectral domain OCT allowed imaging of aspects such as CL and ocular surface relationships, noninvasively, with much higher degrees of axial and lateral resolution for wider fields of view $[3,12]$. Toth et al. [5] using a modified commercial OCT instrument and were able to obtain cross-sections images of the ocular surface covering a wide angle of view from limbus to limbus and beyond with $3-\mu \mathrm{m}$

*Corresponding author. Email: jgmeijome@fisica.uminho.pt 
axial resolution. They also showed examples of visualization of the tear menisci and post-soft lens tear layer and the profile of the edge of different CLs. At present, there is a limitation in the ability of OCT instruments to automatically detect the limits between thin layers such as the epithelium, flap in corneal refractive surgery, Bowman's layer, or the separation between the cornea and CLs. As in many instruments, this process is still done partially or completely by the operator, it is relevant to evaluate inter-observer and inter-session reliability of this procedure.

In this study, we aim to evaluate the intra-session, inter-session, and inter-observer repeatability of manual segmentation of OCT images to extract CL, post-lens tear film, and different corneal layers thicknesses using a commercial high-resolution spectral domain OCT device.

\section{Methods \\ Subjects}

Five healthy young subjects aged 21-25 years (range, $23.34 \pm 1.43$ years) were randomly fitted on their right or left eye with a thick soft CL. Inclusion criteria required that subjects were not taking any ocular or systemic medication, did not wear CLs before the study, and had no ocular or systemic disease. The study was performed in members of the CEORLab staff who gave their written consent to participate following the tenets of the Declaration of Helsinki. Informed consent was obtained from all volunteers before all the interventions, and they also gave their permission to treat their clinical data anonymously for research purposes.

\section{Contact lenses}

The Soft K soft CL (Soflex; Isralens, Ltd, Misgav, Israel) used in this study is made of a nonionic high water content material $(58 \%)$ under the generic name GM3, which is a copolymer composed of glycerolmethacrylate and vinylpyrrolidone. The lens was fitted after a trial to find the best lens-to-cornea relationship under slit-lamp evaluation. The aim was to obtain good centration to warrant that all volunteers had the same part of the lens over the central cornea. Two different fittings were performed, one parallel fit to minimize the separation between the lens and the cornea (Figure 1(A)), and one steep fit by fitting a lens $0.6 \mathrm{~mm}$ steeper than the previous one to create a separation between the lens and the cornea (Figure 2(A)).

\section{Instrumentation}

A high-resolution spectral domain OCT (Copernicus HRSOCT; Optopol Technology SA, Zawiercie, Poland) was used. This device was conceived as a posterior segment high-resolution OCT providing axial resolutions of $3 \mu \mathrm{m}$ using an 850-nm wavelength, but it can be also used for anterior segment viewing through a coupling device, also commercially available. Anterior segment imaging measures counts with an axial resolution of $2.88 \mu \mathrm{m}$ (according to manufacturer). A 5-mm observation field was obtained for all the images. Scanning speed is up to 52,000 A-scans per second according information from manufacturer.

\section{Measuring protocol}

All patients had been awake for at least at least $4 \mathrm{~h}$ before attending the laboratory on that session. All
PARALELL FIT

(A)

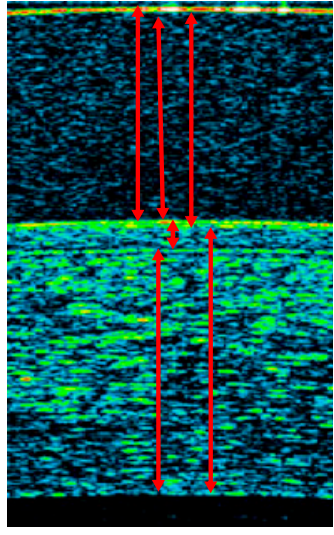

(B)

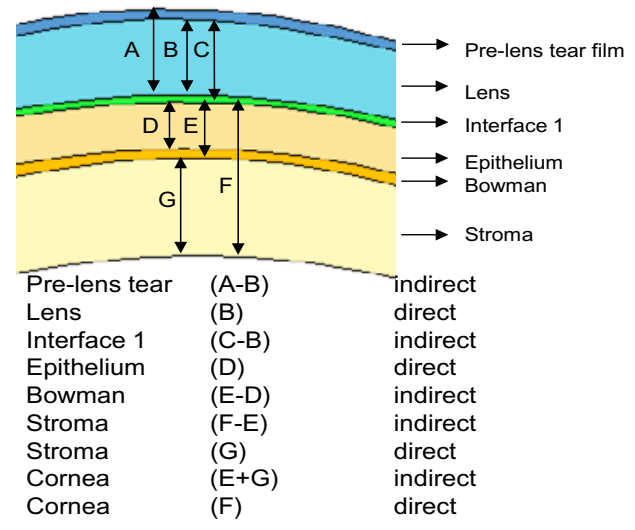

Figure 1. OCT image representing a CL with a parallel fit. Arrows identifying different layers measured $(A)$ and schematic drawing of the same structures at an exaggerated scale to highlight the different parameters obtained $(B)$. (The colour version of this figure is included in the online version of the journal.) 
STEEP FIT

$(A)$

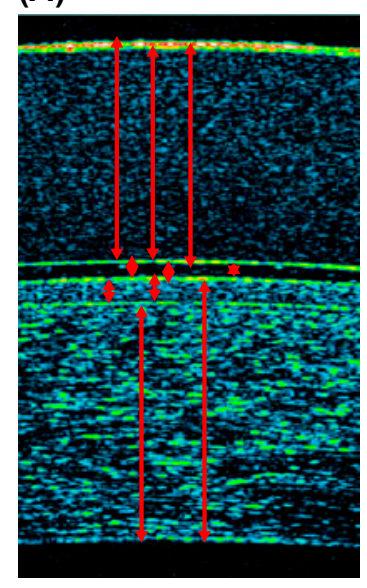

(B)

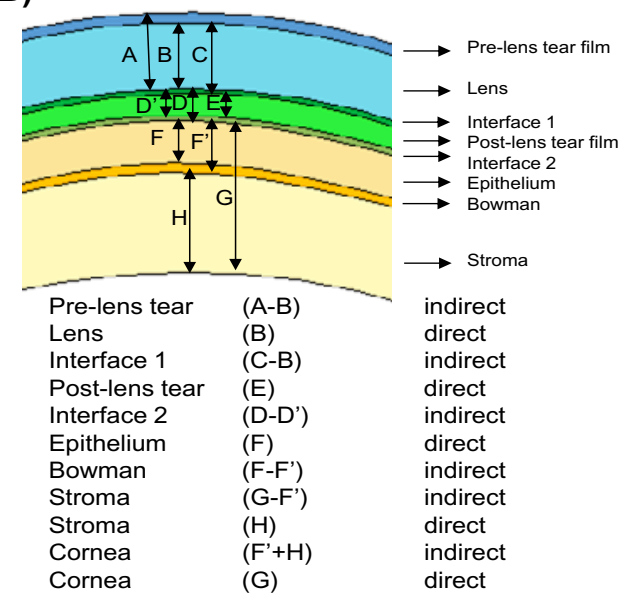

Figure 2. OCT image representing a CL with a steep fit. Arrows identifying different values obtained $(A)$ and schematic drawing of the same structures at an exaggerated scale to highlight the different parameters obtained $(B)$. (The colour version of this figure is included in the online version of the journal.)

volunteers were evaluated between 14:00 and 17:00 $\mathrm{h}$ to minimize diurnal variations in corneal thickness or to avoid potential variations in hydration control during the session $[11,13,14]$. CLs were applied and were allowed to settle for $20 \mathrm{~min}$. Once all alignments were obtained, the volunteer was asked to open the eyes, and with minimal additional adjustments the image was obtained within $3 \mathrm{~s}$ after the last complete blink. To ensure that measurements are reliably obtained at the same corneal location, the specular reflex induced when the incident beam is perpendicular to the corneal apex was used as a reference; at this moment, minimal changes on instrument location were done to get an image free from specular reflex noise.

Then the image was processed and the measures were obtained manually using the instruments software. To improve reliability of measurements, the following procedures were used: (1) the image was magnified to its maximum using the OCT software; (2) the image was kept centered on the visualized area; and (3) the center of the image was used for data collection being coincidental with the corneal location aligned with the instrument during data acquisition. This procedure was maintained for three different acquisitions in the two sessions by both observers.

To determine agreement between different examiners, the examination was done by two observers (observer 1 and observer 2) within $30 \mathrm{~min}$ interval, taking three repeated measures. The three measures from each observer were also used to evaluate the repeatability of measures by each observer. To evaluate the inter-session agreement, the retest was done by user 1 and 2 on the following session at the same time and in the same conditions.
The outcome measures were the thickness of the prelens tear film, the lens, post-lens tear film, epithelium, bowman's layer, stroma, and the whole cornea. For some thin layers (post-lens tear film, Bowman's layer), the thickness was subtracted from the thicker surrounding layers (CL, cornea or epithelium, and stroma, respectively) in order to minimize the potential errors of edge detection when trying to isolate the borders of the thin layer directly. The layers under evaluation as well as the methods used to obtain each (direct or indirect) are illustrated in Figure 1(B) for the parallel fit and in Figure 2(B) for the steep fit.

\section{Statistical analysis}

The data were entered in an MS Excel sheet (Microsoft Inc, Redmond, Wash.) and transferred for analysis to SPSS 21.0 (SPSS Inc, Illinois). Considering the reduced sample, Kruskall-Wallis test was used to evaluate the statistical significance of differences between the three measures obtained within the same session. Comparisons between data from the same observer on different sessions were evaluated using Wilcoxon signed ranks test while differences between observers on session 2 were compared by Mann-Whitney U-test. Spearman's correlation coefficients (CC) and best fit linear equations were computed to assess the correlation between measures between repeated measures obtained in two different sessions. Interclass correlation coefficient (ICC) was also computed to estimate the proportion of variability between subjects compared with overall variability. A $p$-value lower than 0.05 was considered statistical significant. 


\section{Results}

Table 1 presents the average values of thickness averaged from three repeated measures in each session (Session 1 and Session 2) for both observers (Observer 1 and Observer 2). There was a high level of agreement between the measures obtained by each observer on each session. Overall, this agreement was stronger for the thicker layer with higher $\mathrm{CC}$ and ICC and weaker from the thinner layers. However, differences between sessions for the same observer were non-statistically significant for any of the layers and for any observer (Wilcoxon signed ranks test, $p>0.05$ ). A closer observation to the average of individual data from three repeated measures carried out on session 1 by each observer, showed that no statistically significant differences were found either (Kruskall-Wallis, $p>>0.05$ ). No statistically significant differences were observed between the thickness of different layers when obtained from each one of the observers (Table 2) demonstrating an acceptable withinobserver repeatability.

Table 3 shows the comparisons between average values of observer 1 and observer 2 on session 2. There were not significant differences between both for any of the parameters measured, displaying also high correlation, and in ICC for all layers under analysis. Figure 3 illustrates the correlation between direct and indirect measures of total corneal and stromal thicknesses.

\section{Discussion}

Corneal thickness is important to accurately estimate the anatomical characteristics and physiological function of the cornea [15]. At present, full corneal thickness and sub-layer thickness are mainly evaluated in the clinical practice as an indicator of corneal function and health [16]. As an example, the epithelial tissue may become thinner as a result of continued wear of hydrogel CLs, due to overnight wear as occurs during orthokeratology treatment, or in ectatic disorders such as keratoconus [17-19].

The most common approaches to measure the corneal thickness in the clinical setting and in research studies are currently ultrasound (US) pachymetry and optical slit methods such as Orbscan [20,21] Pentacam [21], Sirius [22], or Galilei [23]. However, none of these methods is able to separate the different corneal layers (i.e. epithelium, bowman, and stroma) from the total thickness. Anterior segment OCT is a high-resolution technique that obtains cross-sectional images of the eye in vivo noninvasively. It allows measuring the thickness of different layers of tissues, as well as their overall thickness and the relationship between the ocular surface and CLs [24]. It is also used to evaluate their physiological effect onto the stromal and epithelial thickness [25]. Wirbelauer and colleagues [26] showed that, for clinical purposes, central corneal pachymetry with slit lamp-adapted OCT could be an accurate and reproducible technique with a high degree of agreement compared with US pachymetry. However, such technique would be unable to measure separately the different corneal layers. In this study, the cornea was evaluated in detail using a high-resolution spectral domain OCT to measure the corneal thickness layer by layer in manual mode, assessing reproducibility between different observers in one single session and between two different sessions, as well as the reliability of each observer.

Differences between manual and automatic segmentation of the tissues have been under the scope of previous research [27]. Overall, the automatic segmentation fails to separate the thinner layers or the post-lens tear-film space and bowman layer in most instruments. This is the reason why manual segmentation was chosen for the purposes of this study. Furthermore, both direct and indirect estimates of layer thickness were used. The second strategy might allow to improve repeatability in the detection of some thin layers whose thickness can be obtained by the difference between two other thicker layers. The thicker the layer, the lower the error we might have in measuring it; thus, resulting in an overall better accuracy of the layer we intend to measure.

Previous authors already noticed that the failure of the determination of the corneal layers boundaries was related with the fact of OCT image have a low signal to noise ratio and contains image non-homogeneities; the boundary detection methods are dependent upon the image gradient obtained from the noisy OCT image; and the inner boundaries of the OCT image are indistinct [28]. Increasingly complex algorithms are being applied for such automatic detection $[28,29]$. So there are at least two studies that evaluated accuracy by automatic algorithms segmentation of retinal layers [27] and also corneal layers [29], confirming that the automatic algorithm accurately segmented retinal and corneal layers in normal eyes. Despite this, it has been pointed out that different observers might provide slightly different results, while within clinically acceptable levels of variability [29]. Our results also showed that the repeatability and the average values obtained by different observers might be slightly different, particularly for the thinner layers under evaluation. Despite this, the values obtained are in good agreement with those expected for the given layers in normal eyes [17,30,31] and do also present an acceptable level of agreement between observers and between measuring sessions.

The inter-session repeatability (Table 1) was calculated considering the mean value of three repeated measures of the central corneal layers thickness in each session by each observer. The evaluation of thicker layers as CL, stroma (by indirect and direct evaluation), 


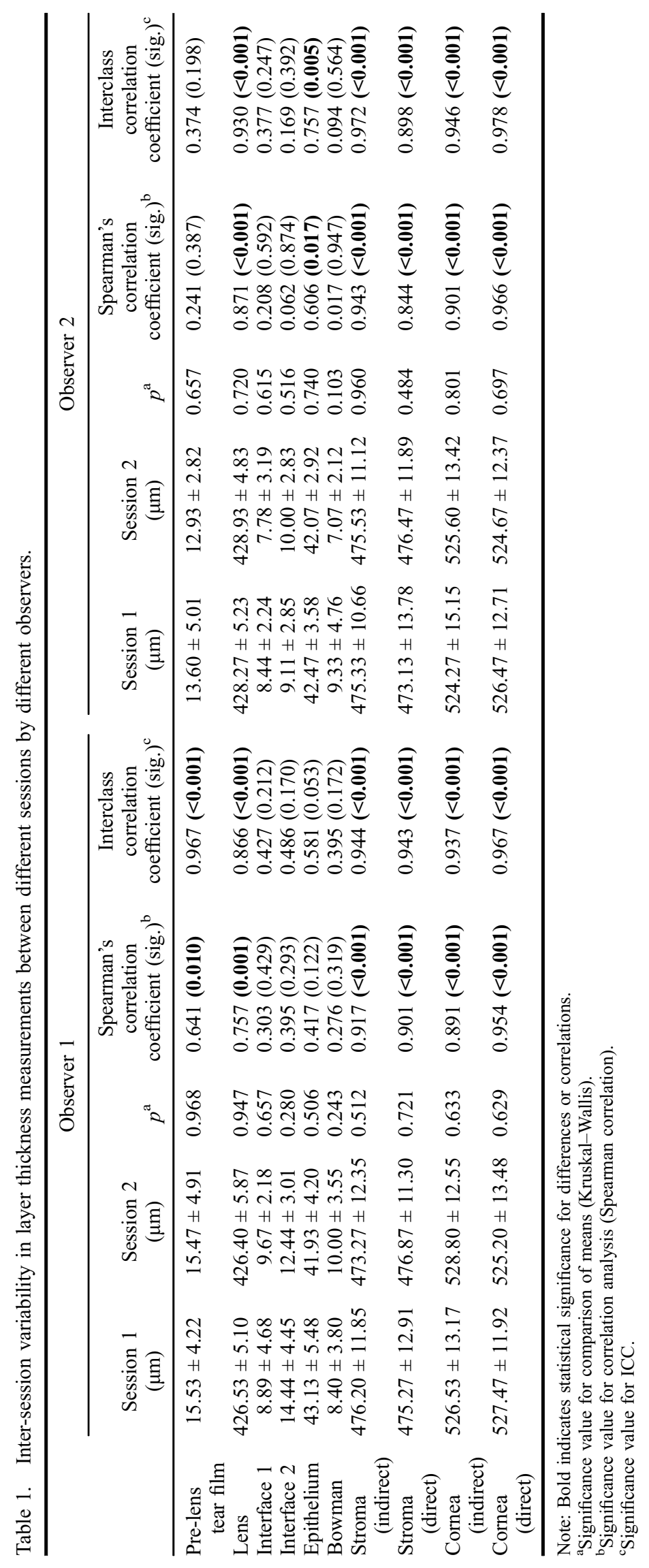


Table 2. Comparison of means among three measurements of layer thickness performed by each observer within the same session.

\begin{tabular}{|c|c|c|c|c|c|c|c|c|}
\hline & \multicolumn{8}{|c|}{ Session 1} \\
\hline & \multicolumn{4}{|c|}{ Observer 1} & \multicolumn{4}{|c|}{ Observer 2} \\
\hline & $\mathrm{m} 1(\mu \mathrm{m})$ & $\mathrm{m} 2(\mu \mathrm{m})$ & $\mathrm{m} 3(\mu \mathrm{m})$ & $p^{\mathrm{a}}$ & $\mathrm{m} 1(\mu \mathrm{m})$ & $\mathrm{m} 2(\mu \mathrm{m})$ & $\mathrm{m} 3(\mu \mathrm{m})$ & $p^{\mathrm{a}}$ \\
\hline $\begin{array}{l}\text { Pre-lens tear } \\
\text { film }\end{array}$ & $15.60 \pm 3.29$ & $13.20 \pm 4.38$ & $17.80 \pm 4.38$ & 0.239 & $15.20 \pm 3.35$ & $11.80 \pm 4.27$ & $13.80 \pm 7.16$ & 0.594 \\
\hline Lens & $427.20 \pm 5.76$ & $427.60 \pm 3.85$ & $424.80 \pm 6.10$ & 0.677 & $426.80 \pm 6.87$ & $429.60 \pm 5.18$ & $428.40 \pm 4.10$ & 0.728 \\
\hline Interface 1 & $8.67 \pm 7.37$ & $8.33 \pm 4.62$ & $9.67 \pm 3.21$ & 0.951 & $7.67 \pm 1.53$ & $9.00 \pm 2.65$ & $8.67 \pm 3.06$ & 0.800 \\
\hline Interface2 & $14.67 \pm 5.03$ & $14.00 \pm 5.29$ & $14.67 \pm 5.03$ & 0.983 & $8.00 \pm 3.46$ & $10.00 \pm 3.46$ & $9.33 \pm 2.31$ & 0.739 \\
\hline Epithelium & $45.80 \pm 5.40$ & $43.80 \pm 3.90$ & $39.80 \pm 6.10$ & 0.221 & $43.00 \pm 2.45$ & $42.20 \pm 4.15$ & $42.20 \pm 4.60$ & 0.931 \\
\hline Bowman & $7.20 \pm 3.90$ & $7.60 \pm 2.61$ & $10.40 \pm 4.56$ & 0.375 & $9.60 \pm 4.77$ & $8.80 \pm 5.22$ & $9.60 \pm 5.37$ & 0.960 \\
\hline $\begin{array}{l}\text { Stroma } \\
\quad \text { (indirect) }\end{array}$ & $479.40 \pm 12.76$ & $475.40 \pm 11.78$ & $473.80 \pm 13.01$ & 0.772 & $476.00 \pm 10.91$ & $475.80 \pm 10.45$ & $474.20 \pm 12.93$ & 0.964 \\
\hline $\begin{array}{l}\text { Stroma } \\
\quad \text { (direct) }\end{array}$ & $473.80 \pm 15.40$ & $475.00 \pm 13.11$ & $477.00 \pm 12.96$ & 0.935 & $473.80 \pm 14.25$ & $473.00 \pm 15.23$ & $472.60 \pm 15.13$ & 0.992 \\
\hline $\begin{array}{l}\text { Cornea } \\
\quad \text { (indirect) }\end{array}$ & $524.00 \pm 15.03$ & $526.40 \pm 13.59$ & $529.20 \pm 13.39$ & 0.844 & $524.40 \pm 16.09$ & $524.00 \pm 17.38$ & $524.40 \pm 15.58$ & 0.999 \\
\hline $\begin{array}{l}\text { Cornea } \\
\quad \text { (direct) }\end{array}$ & $529.60 \pm 12.60$ & $526.80 \pm 12.62$ & $526.00 \pm 13.04$ & 0.897 & $526.60 \pm 13.56$ & $526.80 \pm 13.39$ & $526.00 \pm 14.21$ & 0.995 \\
\hline
\end{tabular}

${ }^{a}$ Significance for comparison of means (Kruskial-Wallis).

Table 3. Comparison between observers in determination of layer thicknesses obtained on session 2.

\begin{tabular}{|c|c|c|c|c|c|}
\hline & \multicolumn{5}{|c|}{ (Session 2_Obs 1 vs. Session 2_Obs 2) } \\
\hline & $\begin{array}{c}\text { Obs } 1 \text { \#session } 2 \\
(\mu \mathrm{m})\end{array}$ & $\begin{array}{c}\text { Obs } 2 \text { \#session } 2 \\
(\mu \mathrm{m})\end{array}$ & $p^{\mathrm{a}}$ & $\begin{array}{l}\text { Spearman Rho } \\
(\text { sig. })^{\mathrm{b}}\end{array}$ & $\begin{array}{l}\text { Interclass correlation coefficient } \\
\qquad(\mathrm{sig})^{\mathrm{c}}\end{array}$ \\
\hline Pre-lens tear & $15.47 \pm 4.91$ & $12.93 \pm 2.81$ & 0.094 & $\begin{array}{c}0.473 \\
(0.075)\end{array}$ & $\begin{array}{c}0.520 \\
(0.058)\end{array}$ \\
\hline Lens & $426.40 \pm 5.87$ & $428.93 \pm 4.83$ & 0.207 & $\begin{array}{c}0.823 \\
(<\mathbf{0 . 0 0 1})\end{array}$ & $\begin{array}{c}0.847 \\
(<\mathbf{0 . 0 0 1})\end{array}$ \\
\hline Interface 1 & $9.67 \pm 2.18$ & $7.78 \pm 3.19$ & 0.162 & $\begin{array}{c}0.581 \\
(0.101)\end{array}$ & $\begin{array}{c}0.626 \\
(0.053)\end{array}$ \\
\hline Interface2 & $12.44 \pm 3.00$ & $10.00 \pm 2.83$ & 0.095 & $\begin{array}{c}0.088 \\
(0.821)\end{array}$ & $\begin{array}{c}0.154 \\
(0.596)\end{array}$ \\
\hline $\begin{array}{l}\text { Epithelium } \\
\text { (direct) }\end{array}$ & $41.93 \pm 4.20$ & $42.07 \pm 2.91$ & 0.920 & $\begin{array}{c}0.683 \\
(\mathbf{0 . 0 0 5})\end{array}$ & $\begin{array}{c}0.792 \\
(\mathbf{0 . 0 0 4})\end{array}$ \\
\hline Bowman's layer & $10.00 \pm 3.55$ & $7.07 \pm 2.12$ & 0.010 & $\begin{array}{c}0.684 \\
(\mathbf{0 . 0 0 5})\end{array}$ & $\begin{array}{c}0.579 \\
(\mathbf{0 . 0 0 7})\end{array}$ \\
\hline Stroma (indirect) & $473.27 \pm 12.35$ & $475.53 \pm 11.12$ & 0.601 & $\begin{array}{c}0.985 \\
(<\mathbf{0 . 0 0 1})\end{array}$ & $\begin{array}{c}0.981 \\
(<\mathbf{0 . 0 0 1})\end{array}$ \\
\hline Stroma (direct) & $476.87 \pm 11.30$ & $476.47 \pm 11.89$ & 0.925 & $\begin{array}{c}0.948 \\
(<\mathbf{0 . 0 0 1})\end{array}$ & $\begin{array}{c}0.974 \\
(<\mathbf{0 . 0 0 1})\end{array}$ \\
\hline Cornea (indirect) & $528.80 \pm 12.55$ & $525.60 \pm 13.42$ & 0.506 & $\begin{array}{c}0.925 \\
(<\mathbf{0 . 0 0 1})\end{array}$ & $\begin{array}{c}0.947 \\
(<\mathbf{0 . 0 0 1})\end{array}$ \\
\hline Cornea (direct) & $525.20 \pm 13.48$ & $524.67 \pm 12.37$ & 0.911 & $\begin{array}{c}0.982 \\
(<\mathbf{0 . 0 0 1})\end{array}$ & $\begin{array}{c}0.990 \\
(<\mathbf{0 . 0 0 1})\end{array}$ \\
\hline
\end{tabular}

Notes: Bold indicates statistical significance for differences or correlations.

${ }^{\text {a}}$ Significance for comparison of means (Kruskal-Wallis).

${ }^{\mathrm{b}}$ Significance value for correlation analysis (Spearman correlation).

${ }^{\mathrm{c}}$ Significance value for ICC.

and total cornea (by indirect and direct evaluation) showed strong inter-session correlation values (Pearson's CC and ICC) above 0.80 being also statistically significant, revealing that it is reliable to compare thickness values of the structures under evaluation at different sessions. Muscat et al. [32] studied the inter-session reproducibility of total corneal thickness $(\mathrm{ICC}=0.979)$ and it could be seen from their report that the mean difference between different sessions was in average about $4 \mu \mathrm{m}(p>0.05)$. Present results are in agreement with Muscat et al., showing similar values for the differences in corneal thickness obtained from different sessions. 
(A)

Corneal total thickness (direct vs indirect evaluation)

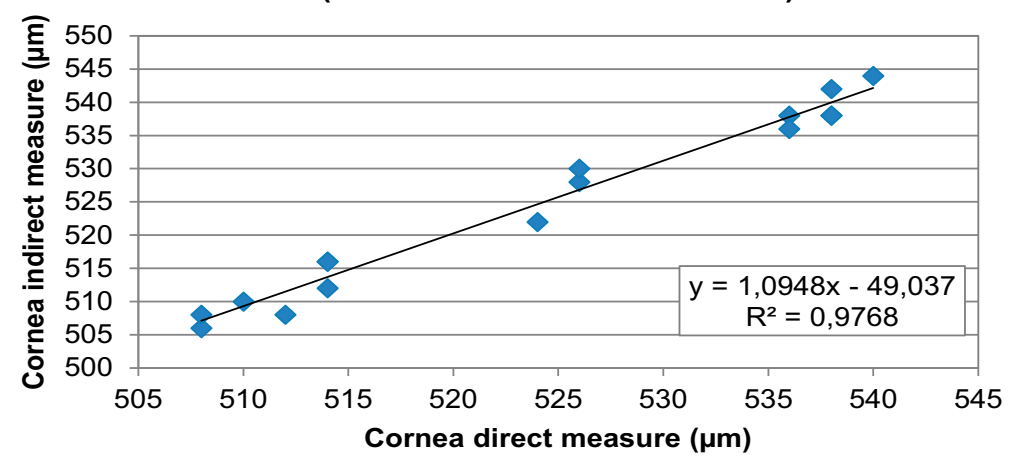

(B) Stromal thickness
(direct vs indirect evaluation)

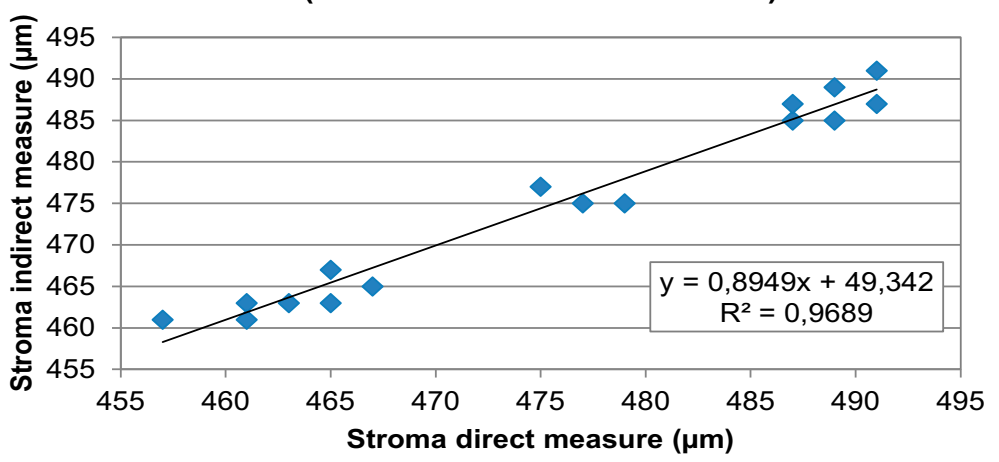

Figure 3. Representation of correlation between total cornea's thickness $(A)$ and total stroma's thickness $(B)$ evaluations obtained under direct and indirect method by OCT. The vertical axis represents the indirect measurements and the horizontal axis shows the direct measures. The bold line is the trendline, and there is also presented the equation that represents the trendline and also the value of determination $\left(r^{2}\right)$. (The colour version of this figure is included in the online version of the journal.)

The intra-session reliability of thickness of different layers was also assessed comparing three repeated measures of each layer for each observer (Table 2). This work did not find differences between the three repeated acquisitions carried out from each layer, neither observer. This suggests that corneal thickness measurements obtained by OCT are robust and relatively free from variability what supports its use at least from the same observer overtime. Similar results have been previously reported for total corneal thickness and epithelial thickness [33]. However, our results suggest the reliability of the epithelium might be a little bit lower when compared with the total corneal thickness, in agreement with the thought that thinner layers are determined manually with higher uncertainty, thought within acceptable limits for repeated measures [25].

Inter-observer repeatability was evaluated with the data from the second session as this was supposed to be the point when both observers were more consistent in their evaluations. A previous investigation [32] showed that the ICC for inter-observers reproducibility was 0.998 for central corneal thickness. This has been quite similar to the values reported by us for the ICC values of the indirect measure of CCT $($ ICC $=0.947)$. The ICC for the direct measure was a little bit lower $(\mathrm{ICC}=0.990)$, confirming that the indirect measure of layers in anterior segment OCT might improve repeatability. It has been observed that the measurements of thinner structures displayed a lower correlation between different observers (Table 3). Bowman's layer thickness obtained was within the range $7-10 \mu \mathrm{m}$, which is slightly lower than those reported in the literature [4,34]. Pre-lens tear thickness values were higher than those reported by other authors $[35,36]$ but the differences observed could be explained by the type of CL used, which was not the same. The intra-observer repeatability for these layers was satisfactory for clinical purposes. Mean epithelial thickness was more reduced than determined by ultrahigh-resolution spectral domain OCT [32], but similar to the values reported in a study that used a modified pachymeter [17].

In summary, the present results suggest that manual segmentation of anterior segment OCT images in CL wearers provides acceptable levels of repeatability 
between observers and between different sessions for the thinner layers, while presenting a high level of repeatability for the thicker layers.

\section{Acknowledgments}

This work was supported by FEDER through the COMPETE Program and by the Portuguese Foundation for Science and Technology (FCT) in the framework of the Strategic Project PEST-C/FIS/UI607/2011. Supported in part by research grants to A. Cerviño from the Universitat de Valencia (UV-AE-20070225), the "Jose Castillejo" Research Grant from the Spanish Ministry of Science and Technology (JC2008-00078) and the Spanish Network for Research in Optometry (SAF2008-01114-E).

\section{Funding}

This work was supported by FEDER through the COMPETE Program and by the Portuguese Foundation for Science and Technology (FCT) in the framework of the Strategic Project PEST-C/FIS/UI607/2011. Supported in part by research grants to A. Cerviño from the Universitat de Valencia (UV-AE20070225), the "Jose Castillejo" Research Grant from the Spanish Ministry of Science and Technology (JC2008-00078) and the Spanish Network for Research in Optometry (SAF2008-01114-E).

\section{ORCID}

José Manuel González-Méijome (iD) http://orcid.org/0000-00019050-4170

\section{References}

[1] Dumbleton, K.A.; Chalmers, R.L.; McNally, J.; Bayer, S.; Fonn, D. Optom. Vis. Sci. 2002, 79, 633-637.

[2] González-Cavada, J.; Corral, O.; Niño, A.; Estrella, M.A.; Fuentes, J.A.; Madrid-Costa, D. J. Optom. 2009, 2, 90-93.

[3] Kaluzny, B.J.; Fojt, W.; Szkulmowska, A.; Bajraszewski, T.; Wojtkowski, M.; Kowalczyk, A. Optom. Vis. Sci. 2007, 84, E1104-E1109.

[4] Wang, J.; Jiao, S.; Ruggeri, M.; Shousha, M.A.; Chen, Q. Eye Cont. Lens 2009, 35, 44-49.

[5] Toth, C.A.; Narayan, D.G.; Boppart, S.A.; Hee, M.R.; Fujimoto, J.G.; Birngruber, R.; Cain, C.P.; DiCarlo, C.D.; Roach, W.P. Arch. Ophthalmol. 1997, 115, 1425-1428.

[6] Bowd, C.; Weinreb, R.N.; Williams, J.M.; Zangwill, L.M. Arch. Ophthalmol. 2000, 118, 22-26.

[7] Izatt, J.A.; Hee, M.R.; Swanson, E.A.; Lin, C.P.; Huang, D.; Schuman, J.S.; Puliafito, C.A.; Fujimoto, J.G. Arch. Ophthalmol. 1994, 112, 1584-1589.

[8] Chen, J.; Lee, L. Clin. Exp. Optom. 2007, 90, 317-335.

[9] Maldonado, M.J.; Ruiz-Oblitas, L.; Munuera, J.M.; Aliseda, D.; García-Layana, A.; Moreno-Montañés, J. Ophthalmology 2000, 107, 81-87.

[10] Feng, Y.; Varikooty, J.; Simpson, T.L. Cornea 2001, 20, $480-483$.
[11] Wang, J.; Fonn, D.; Simpson, T.L.; Jones, L. Am. J. Ophthalmol. 2002, 133, 315-319.

[12] Kaluzny, B.J.; Kaluzny, J.J.; Szkulmowska, A.; Gorczyńska, I.; Szkulmowski, M.; Bajraszewski, T.; Targowski, P.; Kowalczyk, A. Ophthalmic. Physiol. Opt. 2006, 26, 127-132.

[13] Du Toit, R.; Vega, J.A.; Fonn, D.; Simpson, T. Cornea 2003, 22, 205-209.

[14] Handa, T.; Mukuno, K.; Niida, T.; Uozato, H.; Tanaka, S.; Shimizu, K. J. Refract. Surg. 2002, 18, 58-62.

[15] Patel, S.; Marshall, J.; Fitzke, F.W. III J. Refract. Surg. 1995, 11, 100-105.

[16] Feng, Y.; Simpson, T.L. Optom. Vis. Sci. 2005, 82, 416-419.

[17] Pérez, J.G.; Méijome, J.M.; Jalbert, I.; Sweeney, D.F.; Erickson, P. Cornea 2003, 22, 304-307.

[18] Reinstein, D.Z.; Gobbe, M.; Archer, T.J.; Couch, D.; Bloom, B. Optom. Vis. Sci. 2009, 86, E1006-E1014.

[19] Reinstein, D.Z.; Archer, T.J.; Gobbe, M. J. Refract. Surg. 2009, 25, 604-610.

[20] González-Méijome, J.M.; Cerviño, A.; Yebra-Pimentel, E.; Parafita, M.A. J. Cataract Refract. Surg. 2003, 29, $125-132$.

[21] González-Pérez, J.; Gonzalez-Meijome, J.M.; Rodriguez Ares, M.T.; Parafita, M.A. Eye Cont. Lens 2011, 37, $348-353$.

[22] Jorge, J.; Rosado, J.; Diaz-Rey, J.; Gonzalez-Meijome, J. Clin. Ophthalmol. 2013, 7, 417-422.

[23] Demir, S.; Ortak, H.; Yeter, V.; Alim, S.; Sayn, O.; Taş, U.; Sönmez, B. Cornea 2013, 32, 1470-1474.

[24] Gonzalez-Meijome, J.M.; Cerviño, A.; Carracedo, G.; Queiros, A.; Garcia-Lázaro, S.; Ferrer-Blasco, T. Cornea 2010, 29, 1359-1367.

[25] González-Méijome, J.M.; Cerviño, A.; Peixoto-de-Matos, S.C.; Madrid-Costa, D.; Jorge, J.; Ferrer-Blasco, T. Cornea 2012, 31, 633-638.

[26] Wirbelauer, C.; Scholz, C.; Hoerauf, H.; Pham, D.T.; Laqua, H.; Birngruber, R. Am. J. Ophthalmol. 2002, 133, 444-450.

[27] Chiu, S.J.; Li, X.T.; Nicholas, P.; Toth, C.A.; Izatt, J.A.; Farsiu, S. Opt. Express 2010, 18, 19413-19428.

[28] Eichel, J.A.; Misha, A.K.; Clausi, D.A.; Fieguth, P.W.; Bizheva, K.K. Can. Conf. Comput. Robot Vision 2009, 313-320.

[29] LaRocca, F.; Chiu, S.J.; Farsiu, S. Biomed. Opt. Express 2013, 2, 1524-1538.

[30] Reinstein, D.Z.; Archer, T.J.; Gobbe, M.; Silverman, R.H.; Coleman, D.J. J. Refract. Surg. 2009, 25, 776-786.

[31] Tao, A.; Wang, J.; Chen, Q.; Shen, M.; Lu, F.; Dubovy, S.R.; Shousha, M.A. Invest. Ophthalmol. Vis. Sci. 2011, 52, 3901-3907.

[32] Muscat, S.; McKay, N.; Parks, S.; Kemp, E.; Keating, D. Invest. Ophthalmol. Vis. Sci. 2002, 43, 1791-1795.

[33] Sin, S.; Simpson, T.L. Optom. Vis. Sci. 2006, 83, 360-365.

[34] Tao, A.; Wang, J.; Chen, Q.; Shen, M.; Lu, F.; Dubovy, S.R.; Shousha, M.A. Invest. Ophthalmol. Vis. Sci. 2011, 52, 3901-3907.

[35] Nichols, J.J.; Mitchell, G.L.; King-Smith, P.E. Invest. Ophthalmol. Vis. Sci. 2005, 46, 2353-2361.

[36] Wang, J.; Fonn, D.; Simpson, T.L.; Jones, L. Invest. Ophthalmol. Vis. Sci. 2003, 44, 2524-2528. 